\title{
EFFECTIVITY ANALYSIS OF CHOSEN NUMERICAL METHODS FOR SOLUTION OF MECHANICAL SYSTEMS WITH UNCERTAIN PARAMETERS
}

The paper deals with usability and efficiency problem for the chosen solution methods for mechanical systems with structural uncertainties, which are significantly influencing the analysis results and the analysis itself. In the centre of interest will be the chosen approaches and methods. An application of the chosen approaches is presented - the first one, a simple combination of only inf-values or only sup-values; the second one presents full combination of all inf-sup values; the third one uses the optimizing process as a tool for finding out an inf-sup solution and last one is Monte Carlo technique as a comparison tool.

Keywords: uncertain parameters, MATLAB, Monte Carlo, interval arithmetic, optimization

\section{Introduction}

Generally, it is possible to say that each engineering problem encounters uncertainties in various forms, e.g. geometrical parameters, material constants, loads, etc. Many of those uncertainties are based on physical imperfections; the general diversity and complexity of natural phenomena and, of course, our ignorance or inability to precisely describe characteristics of the investigated problem.

Uncertain parameters appear mostly as random variables and thus are described in the terms of stochastic approach. But without the knowledge of the probability density and the nature of distribution we are forced to use another approach, which could describe the parameters with the mentioned restrains and at the same time contain sufficient information about the character of the uncertainty.

Alternately to the use of probability methods we can use imprecise probabilities and the possibility theory, which involves the theory of interval numbers $[2,3,4]$, fuzzy numbers and fuzzy sets $[5,6,9]$. Without the information of the relevance of the data on the interval, we cannot use the fuzzy approach, but we are still able to use the interval approach to describe the uncertain parameters which are considered as unknown but bounded with lower and upper bounds.

Our short study proposes algorithms for modal and spectral interval computations of FE models and their effectivity analysis in view of the input uncertainty degree $(2 \%, 5 \%, 10 \%, 15 \%, 20 \%)$.

\section{Computational methods for interval analysis}

If we want to use interval arithmetic approach, an uncertain number is represented by an interval of real numbers [2, 4]. The interval numbers derived from the experimental data or expert knowledge can then take into account the uncertainties in the model parameters, model inputs etc. Complete information about the uncertainties in the model may be included by this technique and one can demonstrate how these uncertainties are processed by the calculation procedure in MATLAB.

During the solving of the particular tasks using the interval arithmetic application on the solution of numerical mathematics and mechanical problems, the problem known as the overestimate effect is encountered. Its elimination is possible only in the case of meeting the specific assumptions, mainly related to the time efficiency of the computing procedures [1,3]. Considering uncertain parameters in interval form, some solution approaches already used or proposed by the authors are analyzed $[9,11]$. The goal is to present algorithm description and comparison study of the following numerical methods:

- Monte Carlo method (MC) - as a comparison tool,

- a simple combination of only inf-values or only sup-values (COM1),

- a full combination of all inf-sup values (COM2),

- a method which uses an optimization process as a tool for finding out a inf-sup solution (OPT).

Monte Carlo method (MC) is a time consuming but reliable solution. Various combinations of the uncertain parameter deter-

\footnotetext{
* Robert Bednar, Milan Saga, Milan Vasko

Department of Applied Mechanics, Faculty of Mechanical Engineering, University of Zilina, Slovakia,

E-mail: robert.bednar@fstroj.uniza.sk
} 
ministic values are generated and after the subsequent solution in the deterministic sense we obtain a complete set of results processed in an appropriate manner. Infimum and supremum calculation is following

$$
\begin{aligned}
& \inf (F)=\text { min of all results } F\left(p_{i}\right), \\
& \text { where } i=1, \ldots, m \text { and } m \approx 5000 \div 100000 \\
& \sup (F)=\max \text { of all results } F\left(p_{i}\right), \\
& \text { where } i=1, \ldots, m \text { and } m \approx 5000 \div 100000 .
\end{aligned}
$$

Solution evaluation in marginal values of interval parameters (COM1) has its physical meaning for many engineering problems. We consider an approach where the extreme output values are obtained by the application of the extreme parameter values on input. That means that the inf-sup is obtained using the deterministic analysis for inf or sup of input uncertain parameters. Inf-sup calculation is

$$
\begin{aligned}
& \inf (F)=\min \text { of }[F(\underline{p}), F(\bar{p})] \text { and } \\
& \sup (F)=\max \text { of }[F(\underline{p}), F(\bar{p})] .
\end{aligned}
$$

Solution evaluation for all marginal values of interval parameters (COM2) which is also based on the set of the deterministic analyses appears as the more suitable one. The marginal interval parameter values are considered again but the inf and sup values are also combined. The method provides satisfying results and can be marked as reliable, even if there is still a doubt about the existence of the extreme solution for the uncertain parameter inner values. Solution for two interval numbers $p_{1}=\left\langle a_{1} b_{1}\right\rangle$ and $p_{2}=\left\langle a_{2} b_{2}\right\rangle$ may be found by this computational way

$$
\begin{aligned}
& \inf (F)=\min \text { of } F\left[\left(a_{1} a_{2}\right), F\left(a_{1} b_{2}\right), F\left(b_{1} a_{2}\right), F\left(b_{1} b_{2}\right)\right] \\
& \sup (F)=\max \text { of } F\left[\left(a_{1} a_{2}\right), F\left(a_{1} b_{2}\right), F\left(b_{1} a_{2}\right), F\left(b_{1} b_{2}\right)\right]
\end{aligned}
$$

The method of the inf and sup solution using the optimization techniques (OPT) is proposed by the authors as an alternative to the first and to the third method. It should eliminate a big amount of analyses in the first method and also eliminates the problem with the possibility of the inf and sup existence inside of the interval parameters for the deterministic values. Computational process for two interval numbers $p_{1}$ and $p_{2}$ may be found as follows

$$
\begin{aligned}
& \inf (F)=F\left(\boldsymbol{p}_{O P T}\right), \text { i.e. find } \boldsymbol{p}_{O P T} \text { so that } F\left(\boldsymbol{p}_{O P T}\right) \rightarrow \min , \\
& \sup (F)=F\left(\boldsymbol{p}_{O P T}\right), \text { i.e. find } \boldsymbol{p}_{O P T} \text { so that } F\left(\boldsymbol{p}_{O P T}\right) \rightarrow \max ,
\end{aligned}
$$

\section{Interval analysis of a damped computational model}

A numerical analysis of the damped computational model (Fig. 1) with uncertain parameters (damping parameter, stiffness parameter, etc.) in interval form will be presented. The interval modalspectral analysis in the range of the first three eigen shapes was performed. The mechanical system represents half of a vehicle under vertical excitation of the front and rear axle.

It is assumed that the model is linearized about the operating state and that the coordinates $z_{1 V}(t), z_{1 H}(t), z_{2}(t), \varphi_{2}(t)$ and $z_{3}(t)$ are measured from the equilibrium state [3]. The coordinates $z_{2 V}(t)$ and $z_{2 H}(t)$ can be described as functions of the geometry of the model and the coordinates $z_{2}(t)$ and $\varphi_{2}(t)$.

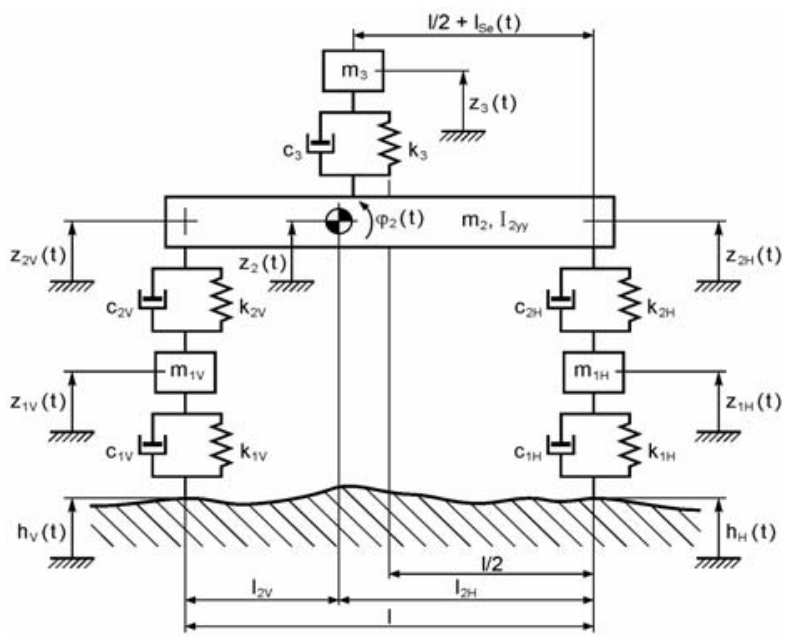

Fig. 1 5-DOF spring-mass-dashpot system

\section{Mathematical model}

As mentioned in previous section, the state space model for the mathematical description is used [7, 8]. The state vector $\boldsymbol{x}(t)$ and the input vector $\boldsymbol{u}(t)$ for the two wheels of the system can be given as

$$
\begin{gathered}
\boldsymbol{x}(t)=\left[z_{1 V}(t) z_{1 H}(t) z_{2}(t) \varphi_{2}(t) z_{3}(t) \dot{z}_{1 V}(t) \dot{z}_{1 H}(t)\right. \\
\left.\dot{z}_{2}(t) \dot{\varphi}_{2}(t) \dot{z}_{3}(t)\right]^{T} \text { and } \boldsymbol{u}(t)=\left[\begin{array}{l}
h_{V}(t) \\
h_{H}(t) \\
\dot{h}_{V}(t) \\
\dot{h}_{H}(t)
\end{array}\right] .
\end{gathered}
$$

For easier representation, the state matrix $\boldsymbol{A}$ of the given system can be decomposed in four sub matrices, i.e.,

$$
A=\left[\begin{array}{cc}
0 & I \\
-M_{S}^{-1} K_{S} & -M_{S}^{-1} C_{S}
\end{array}\right] .
$$

The matrix $\mathbf{0}$ is a 5-by-5 zero matrix and $\boldsymbol{I}$ represents a 5-by5 identity matrix. The mass matrix $\boldsymbol{M}_{\mathrm{s}}$ has five entries along the main diagonal which are not zero

$$
M_{S}=\left[\begin{array}{ccccc}
m_{1 V} & 0 & 0 & 0 & 0 \\
0 & m_{1 H} & 0 & 0 & 0 \\
0 & 0 & m_{2} & 0 & 0 \\
0 & 0 & 0 & I_{2,} & 0 \\
0 & 0 & 0 & 0 & m_{3}
\end{array}\right] .
$$

The stiffness matrix $\boldsymbol{K}_{s}$, a 5-by-5 matrix consisting of the corresponding stiffness coefficients 


\section{COMMNICOIIONS}

$$
K_{S}=\left[\begin{array}{ccccc}
k_{1 V}+k_{2 V} & 0 & -k_{2 V} & k_{2 V} l_{2 V} & 0 \\
0 & k_{1 H}+k_{2 H} & -k_{2 H} & -k_{2 H} l_{2 H} & 0 \\
-k_{2 V} & -k_{2 H} & k_{2 H}+k_{2 V}+k_{3} & k_{3.4} & -k_{3} \\
k_{2 V} l_{2 V} & -k_{2 H} l_{2 H} & k_{4.3} & k_{4.4} & -k_{3}\left(l / 2-l_{2 V}-l_{S e}\right) \\
0 & 0 & -k_{3} & -k_{3}\left(l / 2-l_{2 V}-l_{S e}\right) & k_{3}
\end{array}\right] \text {. }
$$

with

$$
\begin{aligned}
& k_{3,4}=k_{3}\left(l / 2-l_{2 V}-l_{S e}\right) \\
& k_{4,3}=k_{3}\left(l / 2-l_{2 V}-l_{S e}\right) \\
& k_{4,4}=k_{3}\left(l / 2-l_{2 V}-l_{S e}\right)^{2}+k_{2 H} l_{2 H}^{2}+k_{2 V} l_{2 V}^{2}
\end{aligned}
$$

Similar to the stiffness matrix $\boldsymbol{K}_{S}$, the damping coefficient matrix $C_{S}$, also a 5-by-5 matrix is given as

\section{Solving of truss structure with interval parameters}

Considering different uncertain parameters the numerical interval stress-strain study of a three-dimensional truss structure (Fig. 5) was performed.

As the interval uncertain parameters were the cross-sections of the trusses considered. Because of the computation memory and time demands, fifty one bars were split into 7 cross-sectional groups (Fig. 6) [10]. All other parameters were considered as certain.

$$
C_{S}=\left[\begin{array}{ccccc}
c_{1 V}+c_{2 V} & 0 & -c_{2 V} & c_{2 V} l_{2 V} & 0 \\
0 & c_{1 H}+c_{2 H} & -c_{2 H} & -c_{2 H} l_{2 H} & 0 \\
-c_{2 V} & -c_{2 H} & c_{2 H}+c_{2 V}+c_{3} & c_{3.4} & -c_{3} \\
c_{2 V} l_{2 V} & -c_{2 H} l_{2 H} & c_{4.3} & c_{4.4} & -c_{3}\left(l / 2-l_{2 V}-l_{S e}\right) \\
0 & 0 & -c_{3} & -c_{3}\left(l / 2-l_{2 V}-l_{S e}\right) & c_{3}
\end{array}\right] .
$$

with

$$
\begin{aligned}
& c_{3,4}=c_{3}\left(l / 2-l_{2 V}-l_{S e}\right) \\
& c_{4,3}=c_{3}\left(l / 2-l_{2 V}-l_{S e}\right) \\
& c_{4,4}=c_{3}\left(l / 2-l_{2 V}-l_{S e}\right)^{2}+c_{2 H} l_{2 H}^{2}+c_{2 V} l_{2 V}^{2}
\end{aligned}
$$

Percentage variances for real and imaginary parts of $1^{\text {st }}, 2^{\text {nd }}$ and $3^{\text {rd }}$ eigenvalues are shown on Figs. 2-4. The MC, COM2 and OPT methods were used for the interval modal-spectral analysis.
Certain parameters: $E=2 \cdot 10^{11} \mathrm{~Pa}, \mu=0.3, \rho=7800 \mathrm{~kg} \cdot \mathrm{m}^{-3}$,

$$
\delta=10^{-5} \text {. }
$$

Uncertain parameters: $x f=[0.02,0.05,0.10,0.20]$,

$$
\begin{aligned}
& A_{1}=3500 \cdot 10^{-6} \cdot\left(1+x f_{i}\right) \mathrm{m}^{2}, \\
& A_{2}=3000 \cdot 10^{-6} \cdot\left(1+x f_{i}\right) \mathrm{m}^{2}, \\
& A_{3}=2500 \cdot 10^{-6} \cdot\left(1+x f_{i}\right) \mathrm{m}^{2}, \\
& A_{4}=2000 \cdot 10^{-6} \cdot\left(1+x f_{i}\right) \mathrm{m}^{2}, \\
& A_{5}=1800 \cdot 10^{-6} \cdot\left(1+x f_{i}\right) \mathrm{m}^{2}, \\
& A_{6}=1500 \cdot 10^{-6} \cdot\left(1+x f_{i}\right) \mathrm{m}^{2}, \\
& A_{7}=1000 \cdot 10^{-6} \cdot\left(1+x f_{i}\right) \mathrm{m}^{2} .
\end{aligned}
$$

\begin{tabular}{|l|l|l|l|}
\hline \multicolumn{3}{|c|}{ Uncertain parameters } & Certain \\
\hline \multicolumn{1}{|c|}{ Mass parameters } & \multicolumn{1}{|c|}{ Stiffness constants } & Damping coefficients & Distances \\
\hline$m_{1 V}=45 \cdot\left(1+x f_{i}\right) \mathrm{kg}$ & $k_{1 V}=230 \cdot 10^{3} \cdot\left(1+x f_{i}\right) \mathrm{N} / \mathrm{m}$ & $c_{1 V}=46 \cdot\left(1+x f_{i}\right) \mathrm{Ns} / \mathrm{m}$ & $l_{2 V}=1.8 \mathrm{~m}$ \\
$m_{2}=632.5 \cdot\left(1+x f_{i}\right) \mathrm{kg}$ & $k_{1 H}=230 \cdot 10^{3} \cdot\left(1+x f_{i}\right) \mathrm{N} / \mathrm{m}$ & $c_{1 H}=5 \cdot\left(1+x f_{i}\right) \mathrm{Ns} / \mathrm{m}$ & $l_{2 H}=2.2 \mathrm{~m}$ \\
$m_{1 H}=37 \cdot\left(1+x f_{i}\right) \mathrm{kg}$ & $k_{2 V}=22.6 \cdot 10^{3} \cdot\left(1+x f_{i}\right) \mathrm{N} / \mathrm{m}$ & $c_{2 V}=1900 \cdot\left(1+x f_{i}\right) \mathrm{Ns} / \mathrm{m}$ & $l_{S e}=2 \mathrm{~m}$ \\
$m_{3}=28 \cdot\left(1+x f_{i}\right) \mathrm{kg}$ & $k_{2 H}=20 \cdot 10^{3} \cdot\left(1+x f_{i}\right) \mathrm{N} / \mathrm{m}$ & $c_{2 H}=1900 \cdot\left(1+x f_{i}\right) \mathrm{Ns} / \mathrm{m}$ & $l=4 \mathrm{~m}$ \\
$I_{2 y y}=773.5 \cdot\left(1+x f_{i}\right) \mathrm{kg} \cdot \mathrm{m}^{2}$ & $k_{3}=9.9 \cdot 10^{3} \cdot\left(1+x f_{i}\right) \mathrm{N} / \mathrm{m}$ & $c_{3}=260 \cdot\left(1+x f_{i}\right) \mathrm{Ns} / \mathrm{m}$ & \\
\hline
\end{tabular}

where $x f=[0.02,0.05,0.08,0.10,0.12,0.15,0.18,0.20]$. 

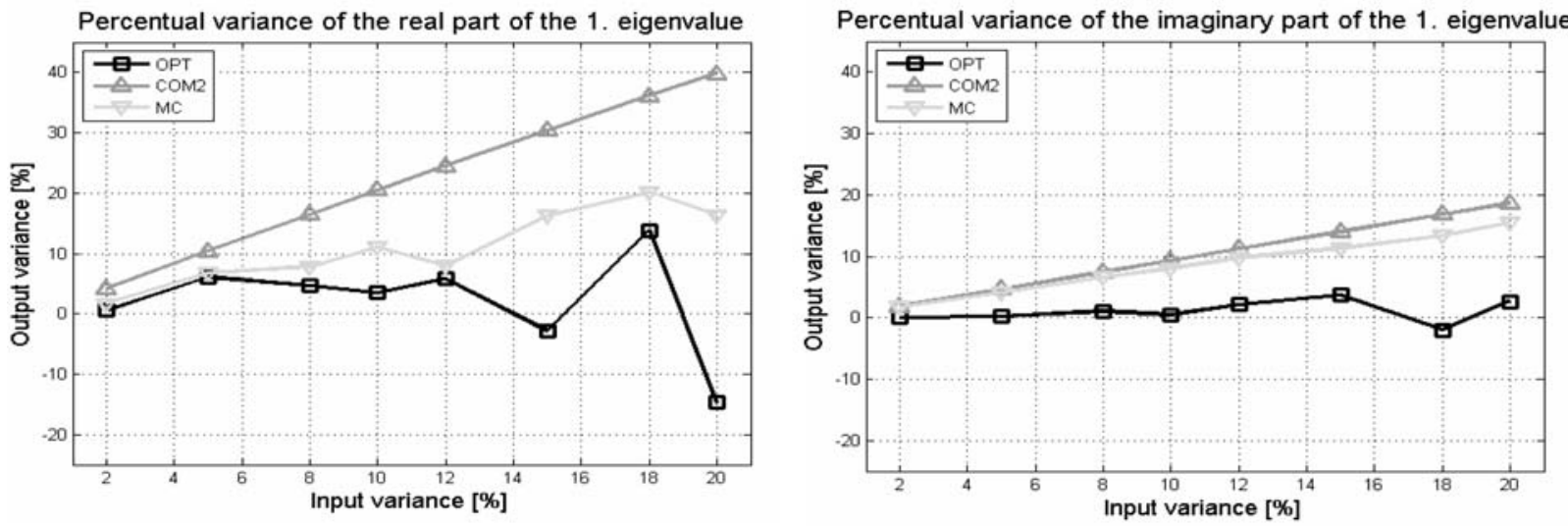

Fig. 2 Percentage variance of the $I^{\text {st }}$ eigenvalue
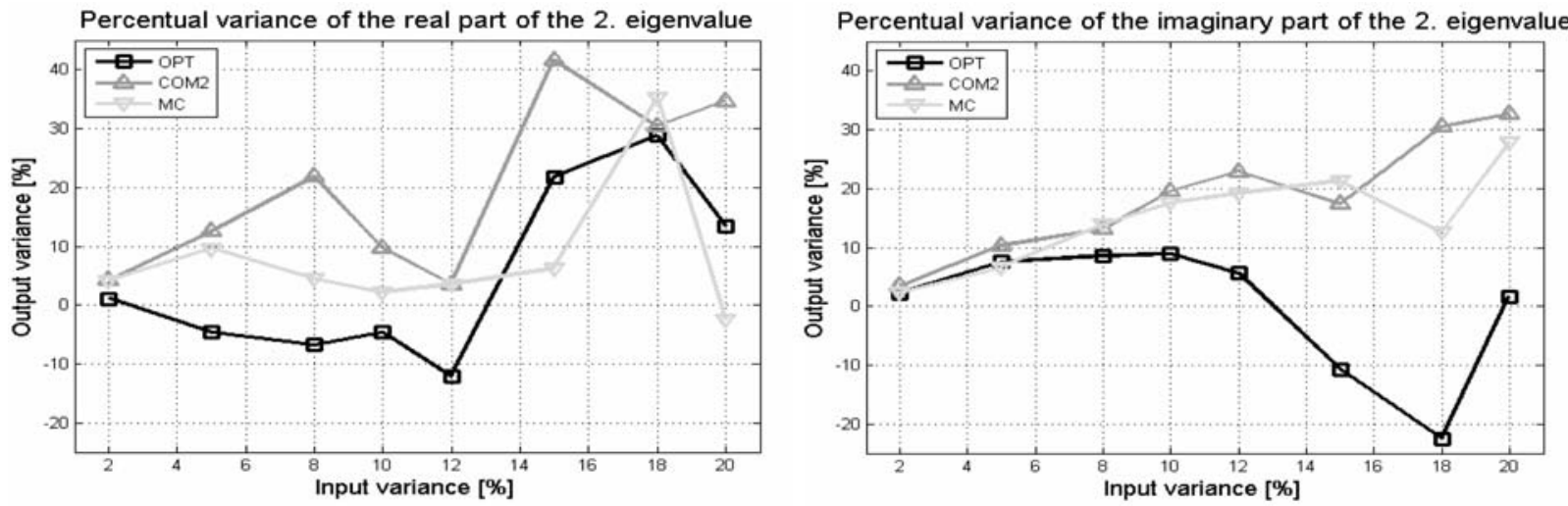

Fig. 3 Percentage variance of the $2^{\text {nd }}$ eigenvalue
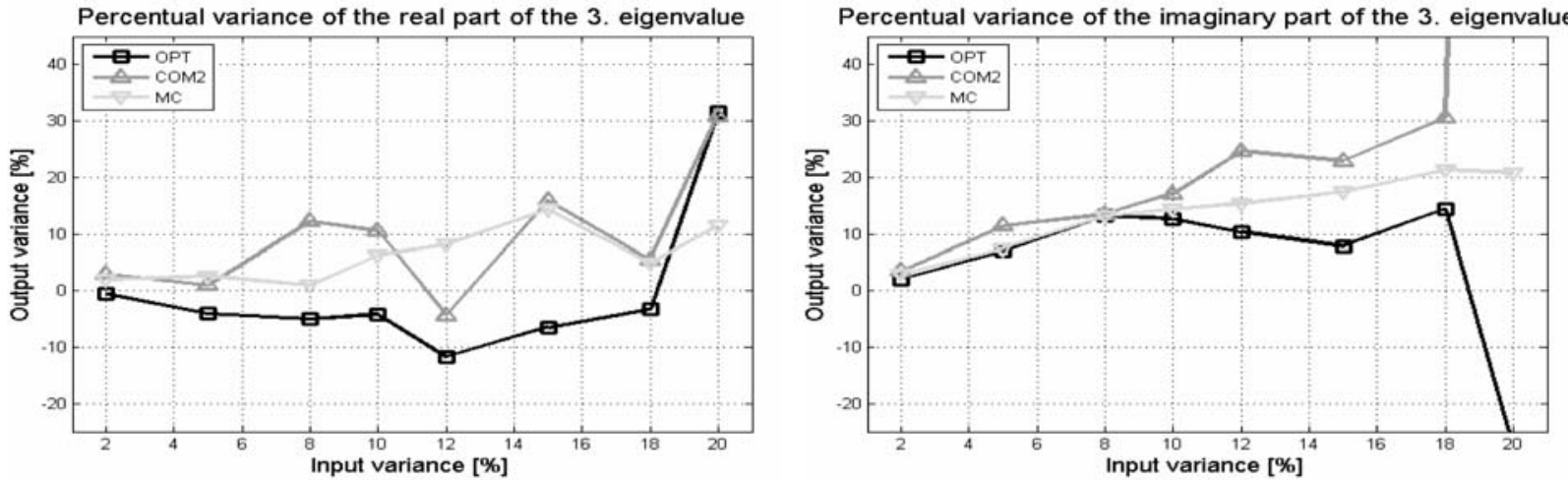

Fig. 4 Percentage variance of the $3^{\text {rd }}$ eigenvalue 


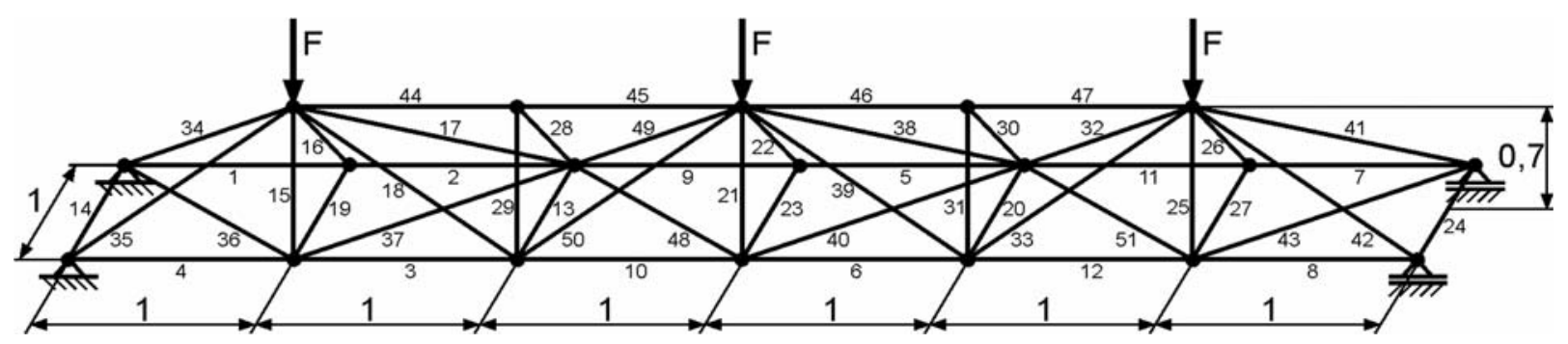

Fig. 5 Analyzed truss structure, dimensions in [m]

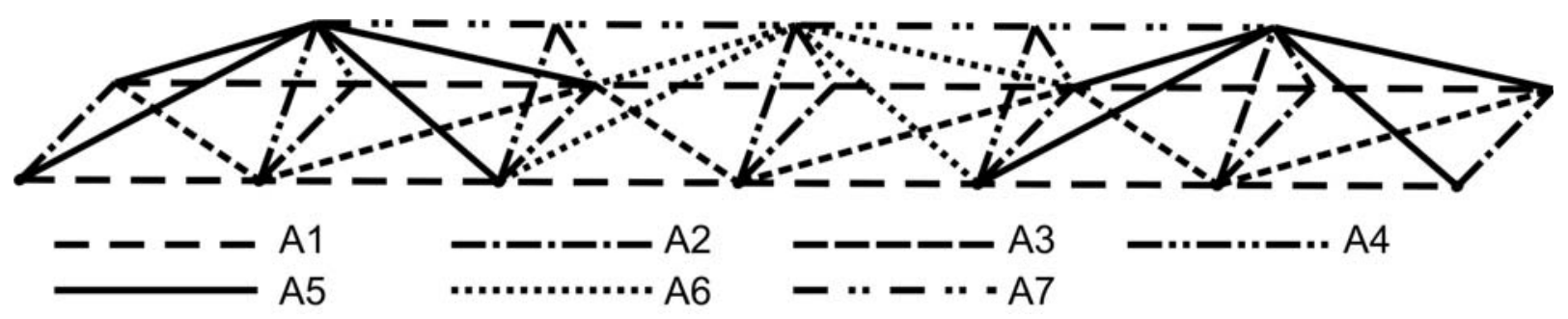

Fig. 6 Truss structure split into 7 cross-sectional groups

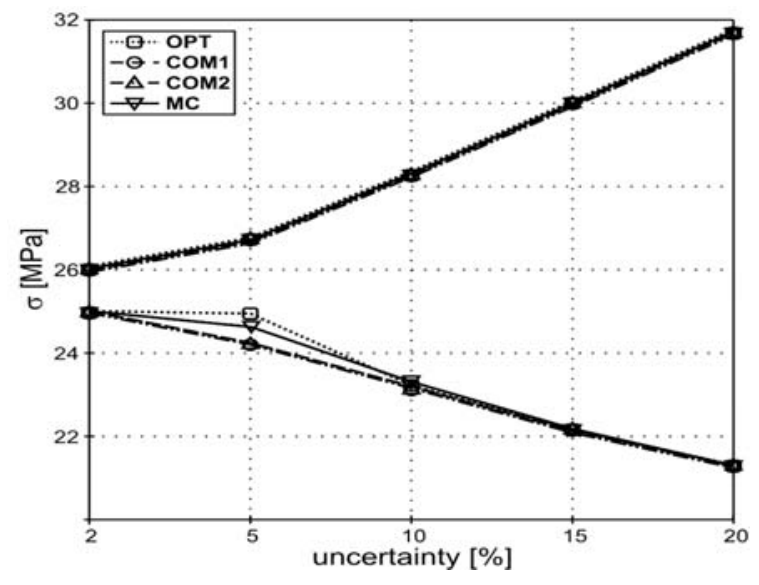

Fig. 7 Stress solution on beam No. 5

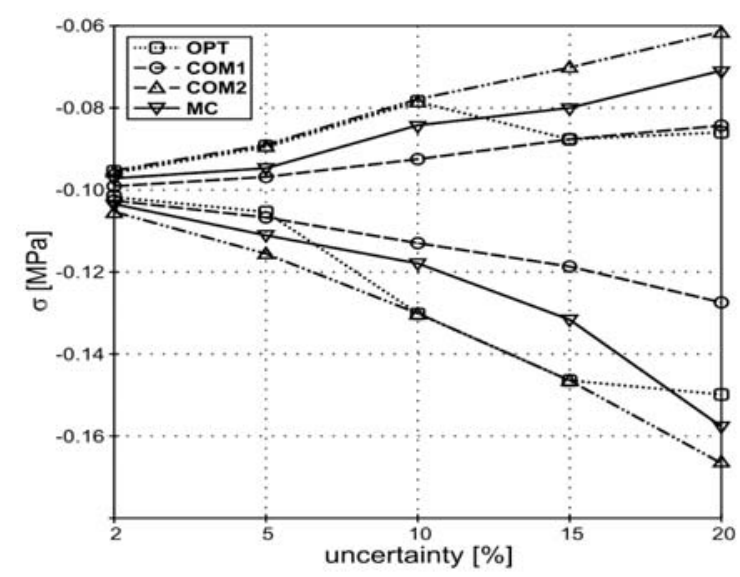

Fig. 9 Stress solution on beam No. 36

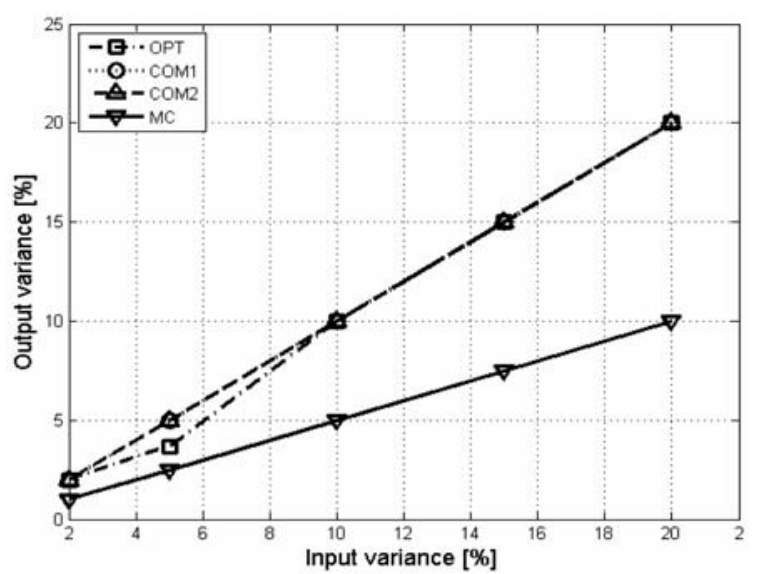

Fig. 8 Percentage variance on beam No. 5

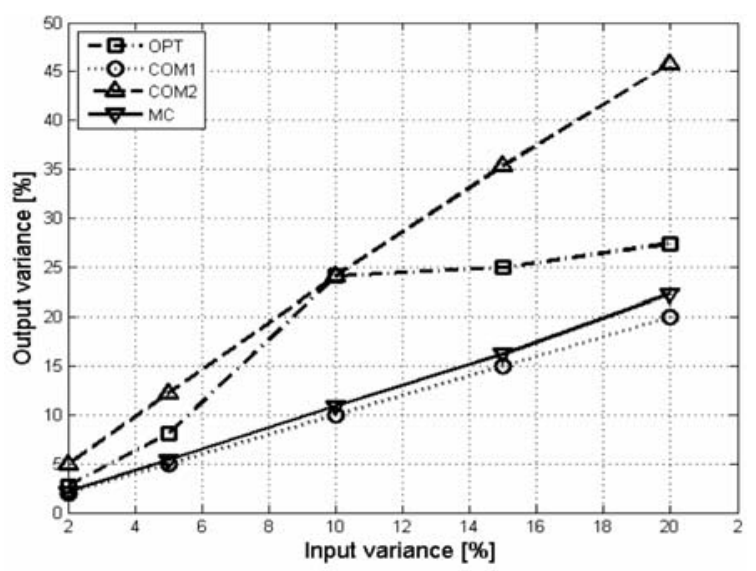

Fig. 10 Percentage variance on beam No. 36 


\begin{tabular}{|c|c|c|c|c|c|}
\hline Uncertainty & Bar No. & MC & OPT & COM1 & COM2 \\
\hline \multirow{2}{*}{$2 \%$} & 5 & $\langle 25.01026 .030\rangle$ & $\langle 25.01026 .030\rangle$ & $\langle 25.01026 .030\rangle$ & $\langle 25.01026 .030\rangle$ \\
\cline { 2 - 6 } & 36 & $\langle-0.104-0.097\rangle$ & $\langle-0.102-0.096\rangle$ & $\langle-0.103-0.099\rangle$ & $\langle-0.106-0.096\rangle$ \\
\hline \multirow{2}{*}{$5 \%$} & 5 & $\langle 24.75426 .853\rangle$ & $\langle 24.94626 .853\rangle$ & $\langle 24.29526 .853\rangle$ & $\langle 24.29526 .853\rangle$ \\
\cline { 2 - 6 } & 36 & $\langle-0.110-0.095\rangle$ & $\langle-0.106-0.090\rangle$ & $\langle-0.107-0.097\rangle$ & $\langle-0.115-0.090\rangle$ \\
\hline \multirow{2}{*}{$\mathbf{1 0 \%}$} & 5 & $\langle 23.26428 .345\rangle$ & $\langle 23.19128 .345\rangle$ & $\langle 23.19128 .345\rangle$ & $\langle 23.19128 .345\rangle$ \\
\cline { 2 - 6 } & 36 & $\langle-0.118-0.085\rangle$ & $\langle-0.130-0.079\rangle$ & $\langle-0.113-0.092\rangle$ & $\langle-0.130-0.079\rangle$ \\
\hline \multirow{2}{*}{$\mathbf{2 0} \%$} & 5 & $\langle 21.25831 .888\rangle$ & $\langle 21.25831 .888\rangle$ & $\langle 21.25831 .888\rangle$ & $\langle 21.25831 .888\rangle$ \\
\cline { 2 - 6 } & 36 & $\langle-0.158-0.071\rangle$ & $\langle-0.150-0.086\rangle$ & $\langle-0.127-0.085\rangle$ & $\langle-0.167-0.062\rangle$ \\
\hline
\end{tabular}

\section{Conclusion}

The paper presents the interval arithmetic application on structural FE analysis and on a modal and spectral analysis. The interval arithmetic provides a new possibility of the examination of quality and reliability of analyzed objects. In the paper authors investigated possibilities of the stress-strain solution of models with interval cross-sectional areas of the truss structure.

It shows the solution efficiency for solving problems including uncertain parameters with a various width of the interval. The interval arithmetic was chosen as a tool for describing various uncertain characteristics of a damped mechanical model. The solution efficiency for solving problems including uncertain parameters with a various width of the interval is shown.

The presented analyses results can be summarized as follows:
- MC is a sure method for obtaining adequate solution results, with the regard of the amount of analyses needed,

- COM1 method gives satisfactory results and can be described as reliable for this kind of analyses, although doubt arises in the sense of the existence of extreme solution for inner values of uncertain parameters,

- COM2 method provides decent results, but it is limited due to the exponential growth of the analyses number for complicated problems, once again arises doubt in the sense of existence of extreme solution for inner values of uncertain parameters,

- OPT method provides good results and is suitable for complicated problems because it does not need so many analyses as in the cases of the MC or COM2 methods.

\section{Acknowledgement}

The work has been supported by the grant project VEGA 1/0125/09, VEGA 1/0727/10.

\section{References}

[1] VASKO, M., SAGA, M., TOTH, L., JAKUBOVICOVA, L.: Short Comparison Study of the Interval Arithmetic Application and Monte Carlo Simulation. In: Computational Mechanics 2003 : Nectiny, ISBN 80-7082-999-0, pp. 467-474, 2003.

[2] MOORE R., E.: Interval Analysis. Prentice Hall, Englewood Cliffs : New Jersey, 1966.

[3] NEUMAIER, A.: Interval Methods for Systems of Equations. Cambridge University Press : Cambridge, 1990.

[4] ELISHAKOFF, I., DUAN, D.: Application of Mathematical Theory of Interval Analysis to Uncertain Vibrations. In: Proc. of NOISECON'94 : Ft. Lauderdale, pp. 519-524, 1994.

[5] CHEN, S. H., YANG, X. W.: Interval Finite Element Method for Beam Structures. In: Finite Elem. Anal. Des., 34, pp. 75-88, 2000.

[6] ZHANG, H.: Nondeterministic Linear Static Finite Element Analysis: An Interval Approach. School of Civil and Environmental Engineering : Georgia Institute of Technology, 2005.

[7] GERLICI, J., LACK, T.: Methods for Vehicle Vibration Analysis in Time Domain. In: Prace naukowe Politechniki Warszawskiej. Z. 63, Transport 2007 : Publishing House of the Warsaw University of Technology, pp. 71-81. ISSN 1230-9265, 2007.

[8] SEGLA, S.: Dynamic and Quasistatic Analysis of the Elevating Work Platform. In: J. Mechanisms and Manipulators, Vol. 6, Nr. $1,2007$.

[9] SAGA, M., VASKO, M.: Algorithmization of Interval Structural Analysis. In: J. of Engineering, Annals of Faculty of Engineering Hunedoara, Tome VI, 2008, ISSN 1584-2665, pp. 149-160.

[10] VASKO, A.: Image Analysis in Materials Engineering. In: Konferencje, Poland, 2007, Nr 61, pp. 667-670. ISSN 1234-9895.

[11] VASKO, M.: Application of Genetic Algorithms for Solving of Mechanical Systems with Uncertain Parameters. PhD. thesis, Zilina, 2007. 